\title{
Theta waves in treating Auditory Hallucinations using Superconscious meditation.
}

Dr Bheemaiah, Anil Kumar, Vayu Vaidya, Seattle, WA, 98125 USA.

miyawaki@yopmail.com

\begin{abstract}
:
Theta waves, generated in intense 10 minute superconscious meditation has been known to reduce the incidence of auditory hallucinations in schizophrenics, in a study using the Muse Headset, a new era of personal EEG wearables, the author, over a period of 3 weeks has proven the efficacy of superconscious meditation in successfully preventing psychosis in schizophrenia spectrum disorder, in conjunction with stuffed toy based avatar therapy, meditation could work as a replacement for psychiatric medicines, which have harmful side effects and often increase the severity of symptoms, rather than heal them.
\end{abstract}

Keywords: Schizophrenia, Auditory Hallucinations, EEG Wearables, Avatar Therapy, Theta Waves.

\section{What is Schizophrenia?}

The status of schizophrenia as a disorder has been controversial since its original description by Kraepelin and Bleuler.

In particular, a decade of findings on the meta-structure of mental disorders, the development and course of at-risk youth, and genetic epidemiology can be understood as direct challenges to the idea of a specific etiology for the disorder.
Instead of a well-mannered diagnostic entity, schizophrenia and thought disorder more generally delineate a psychosis spectrum linked to a number of other psychiatric outcomes, including, but not limited to, bipolar affective disorder.

Studies of the cognitive impairments associated with the disorder show that a generalized deficit is a prominent behavioral feature of the disorder. "This chapter concludes by noting that spectrum constructs do not preclude 
generating and testing falsifiable hypotheses. The use of a fault tree analysis, as employed in reliability engineering, may be helpful in delineating such hypotheses explicitly. "(MacDonald 2013)

"This is how the Diagnostic and Statistical Manual ( DSM-5 ), a clinical bible created by the American Psychiatric Association (APA), describes schizophrenia: Schizophrenia, 295.90 (F20.9)

A. Two (or more) of the following, each present for a significant portion of time during a 1-month period (or less if successfully treated). At least one of these 3 must be (1), (2), or (3):

1. Delusions.

2. Hallucinations.

3. Disorganized speech (e.g., frequent derailment or incoherence).

4. Grossly disorganized or catatonic 4 behavior.

5. Negative symptoms (i.e., diminished emotional expression or avolition).

B. For a significant portion of the time since the onset of the disturbance, level of functioning 5 in one or more major areas, such as work, interpersonal relations, or self-care, is markedly below the level achieved prior to the onset (or when the onset is in childhood or adolescence, there is failure to achieve expected level of interpersonal, academic, or occupational functioning).

C. Continuous signs of the disturbance persist for at least 6 months. This 6-month period must include at least 1 month of symptoms (or less if successfully treated) that meet Criterion A (i.e., active-phase symptoms) and may include periods of prodomal or residual symptoms. During these prodomal or residual periods, the signs of the disturbance may be manifested by only negative symptoms or by two or more symptoms listed in Criterion A present in an attenuated form (e.g., odd beliefs, unusual perceptual experiences).

D. Schizoaffective disorder and depressive or bipolar disorder with psychotic features have been ruled out because either 1) no major depressive or manic episodes have occurred concurrently with the active-phase symptoms, or 2) if mood episodes have occurred during active-phase symptoms, they have been present for a minority of the total duration of the active and residual periods of the illness.

E. The disturbance is not attributable to the physiological effects of a substance (e.g., a drug of abuse, a medication) or another medical condition. F. If there is a history of autism spectrum disorder or a communication disorder of childhood onset, the additional diagnosis of schizophrenia is made only if prominent delusions or hallucinations, in addition to the other required symptoms of schizophrenia, are also present for at least 1 month (or less if successfully treated)."(Wang 2019)

\section{Auditory Hallucinations, Schizophrenia.}

Auditory Hallucinations is characterized by the patient hearing imagined or 
sometimes real voices, depending on the nature of the society and cultural factors. Auditory hallucinations is normal as a means of collaboration in some communities, while it is termed trespassing and even a form of psychosis in others.

Thematic analysis reveals five essential characteristics: the content of the voices is personally meaningful, the voices have a characterised identity, the person has a relationship with their voices, the experience has a significant impact on the voice-hearer's life, and the experience has a compelling sense of reality.

Implications for treatment interventions include the importance of acknowledging that voices are real, supporting the person to make meaning of who the voices are and what the voices say, and making connections between relationships with voices and relationships with significant others in the person's life.

(Suri 2011; “[No Title]” n.d.)

\section{Medication, Drugs, efficacy,} hallucinations, schizophrenia.

"The prevalence of resistant schizophrenia ranged from 5 to $60 \%$ (Table 1) in the four studies in the literature. Vanelle only found a low rate of $5 \%$ resistance because of too restrictive criteria of resistance corresponding to stages 5 and 6 of Dencker and May defining TRS. The results of the other three studies suggest that an important rate of patients do not derive virtually any benefit of treatment and that the TRS is therefore a true public health problem [7]. Many authors agree on the fact that $1 / 5$ to $1 / 3$ of patients are resistant to treatment "(Dammak 2013) Resistant Schizophrenia, generally calls for alternative approaches, including CBT, MiCBT and SuperConscious Meditation and T.M.

\section{Meditation Therapy in Schizophrenia.}

Meditation especially transcendental and mindfulness has been successfully used in the past two decades to treat a vast range of mental illnesses, ranging from autism spectrum to psychosis spectrum and depression/Bipolar. Much of the published literature consists of meditation practices ranging from loving-kindness meditation, mindfulness, vipassana, transcendental meditation and superconsciousness meditation, including sahaja meditation.(Bricker and Labin 2012; Wahbeh and Nelson 2018; P. D. Silva and De Silva 1995; P. de Silva and de Silva 1989; Kwee 1995; Mahoney 2006; Ellis 2017)

\section{Theta Waves in Kundalini Meditation.}

Increased Theta waves in Kundalini meditation, is demonstrated to decrease auditory hallucinations by $80 \%$ and over a period of three weeks, with at least 10 minute meditation sessions a day, eliminate auditory hallucinations with an efficiency matching computer voice assisted therapy. 
An increase of Theta waves by $40-60 \%$, is part of the therapy and has an annulling effect on auditory hallucinations. An increase of beta waves is also shown to annul some kinds of auditory hallucinations. An increase in beta waves is a normal result of mindfulness meditation.

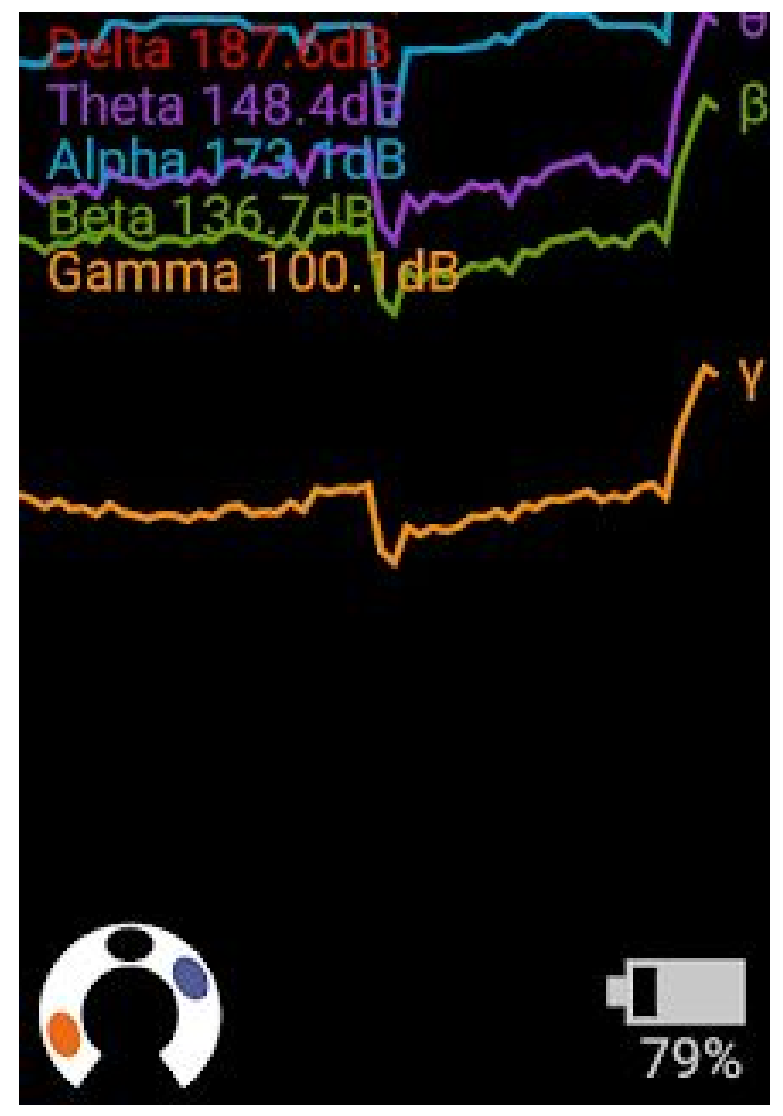

Fig 1: Showing increase of Theta waves to $148.4 \mathrm{~dB}$, decreasing hallucinations by $95 \%$ in subject Dr Bheemaiah, Anil K.

Meditation was performed with the Muse headset and a specialized Android Application to Log EEG waves, performed at Maharishi University of Management, Center for Brain, consciousness and Cognition. Subject: Dr Bheemaiah, Anil Kumar. Measurements are from TP9, AF7, AF8, and TP10, electrodes and two ear electrodes. The average power level and power density are plotted with time for an algorithmic measure of absolute power spectrum level and beta/alpha and delta/theta ratio. These ratios indicate levels of meditation by a quantitative index and a trigger for a bird chirp.

MUSE (RRID: SCR_014418)

It is a portable scalp electroencephalography (EEG) system that is used to measure brain activity. It is battery powered and has four active electrodes located at 10-20 coordinated TP9, AF7, AF8, and TP10, a common mode referenced, and a driven right leg. It includes an accelerometer and gyroscope, and works with desktop and mobile EEG acquisition and visualization software.

\section{Conclusions.}

We have clinically demonstrated the decrease in auditory hallucination, by the increased theta wave density with Superconscious Meditation.

Future work would entail investigating the increase in Beta waves and its correlation with increased Theta waves.

\section{References.}

Bricker, David, and Miriam Labin. 2012.

"Teaching Mindfulness Meditation within a Schema Therapy Framework." The Wiley-Blackwell Handbook of Schema Therapy. https://doi.org/10.1002/978111996283 $0 . \operatorname{ch} 18$.

Dammak, Mohamed. 2013. "Mental Disorders 
- Theoretical and Empirical

Perspectives." In Mental Disorders -

Theoretical and Empirical Perspectives. IntechOpen.

Ellis, Albert. 2017. "The Place of Meditation in

Cognitive-Behavior Therapy and

Rational-Emotive Therapy." Meditation.

https://doi.org/10.4324/978020378584

3-78.

Kwee, Maurits. 1995. "Wherever You Go,

There You Are: Mindfulness Meditation

in Everyday Life." Behaviour Research

and Therapy.

https://doi.org/10.1016/0005-7967(95

)90133-7.

MacDonald, Angus W. 2013. "What Kind of a

Thing Is Schizophrenia?" Schizophrenia.

https://doi.org/10.7551/mitpress/9780

262019620.003.0002.

Mahoney, Anne. 2006. "Meditation in

Therapy." PsycEXTRA Dataset.

https://doi.org/10.1037/e716442007-0

07.

Muse Research:

DOI:10.1371/journal.pone.0130129

"[No Title]." n.d. Accessed August 20, 2019. https://www.tandfonline.com/doi/full/

$10.1080 / 17522431003615622$ ?src $=$ recs ys.

Silva, Padmal de, and Padmal de Silva. 1989.

"The Psychology of Meditation."

Behaviour Research and Therapy.

https://doi.org/10.1016/0005-7967(89

)90022-3.

Silva, P. De, and P. De Silva. 1995.

"Perspectives on Relaxation and

Meditation." Behaviour Research and

Therapy.

https://doi.org/10.1016/0005-7967(95

)90164-7.

Suri, Rochelle. 2011. "Meaningful Voices: A

Phenomenological Exploration of

Auditory Hallucinations in Individuals

With Schizophrenia." PsycEXTRA

Dataset.

https://doi.org/10.1037/e659422011-0

01.

Wahbeh, Helané, and Melissa Nelson. 2018.
"iRest Meditation for Older Adults with Depression Symptoms: A Pilot Study." International Journal of Yoga Therapy. https://doi.org/10.17761/2019-00036. Wang, Esmé Weijun. 2019. The Collected Schizophrenias: Essays. Graywolf Press. 